\title{
Routine endotracheal cultures for the prediction of sepsis in ventilated babies
}

\author{
T A SLAGLE, E M BIFANO, J W WOLF, AND S J GROSS \\ Department of Pediatrics, Health Science Center at Syracuse, New York, USA
}

\begin{abstract}
SUMMARY Serial cultures of endotracheal tube aspirates were carried out in 94 neonates who were intubated and had been ventilated for longer than one week. A similar change in bacterial colonisation with duration of ventilation was seen in infants who subsequently developed sepsis and those who did not. In both groups, 177 aspirates (more than $90 \%$ ) obtained during the first week of ventilation were sterile. Thereafter, colonisation with mixed Gram positive flora emerged followed by growth of Gram negative bacilli. For the infants who developed sepsis, the correlation between pathogens isolated from blood during the episodes of sepsis and those from previous endotracheal tube isolates was poor; in only five of the 26 cases of sepsis $(19 \%)$ was the same single strain of organism isolated from culture of the blood and of the endotracheal tube aspirate. Other markers of infection such as endotracheal tube aspirate white blood cell counts and changes in bacterial flora were not useful in predicting systemic infection. Routine surveillance cultures of endotracheal tube aspirates in ventilated infants are not helpful in predicting the pathogens that are isolated from the blood during episodes of sepsis.
\end{abstract}

Hospital acquired infection is an important cause of mortality and morbidity among infants in neonatal intensive care units. Surveillance cultures are performed routinely in many units to monitor infants at risk of sepsis. ${ }^{12}$ For example, cultures of aspirates obtained through endotracheal tubes from ventilated infants are monitored on the assumption that the organisms that are colonising the respiratory tract will be the same pathogens as those isolated from the blood during episodes of sepsis. Support for this practice comes from studies showing that in infants who develop sepsis at birth, the same bacterial pathogens can be recovered from cultures of blood and endotracheal tube aspirates. ${ }^{3-5}$ The value of serial cultures of endotracheal tube aspirates, however, in determining the aetiology of sepsis in infants who remain intubated for longer periods of time has not been proved. Furthermore, the pattern of organisms colonising the endotracheal tube in ventilated infants after the first week of life has not been elucidated.

This study was designed to define the pattern of respiratory tract colonisation in neonates intubated and ventilated for longer than one week and to assess the value of serial cultures of endotracheal tube aspirates in predicting pathogens isolated during episodes of sepsis.

\section{Patients and methods}

The study population was selected from the 657 neonates born between 1 January 1985 and 31 December 1985 who were admitted to the neonatal intensive care unit at Crouse Irving Memorial Hospital. Ninety four of the 657 neonates $(14 \%)$ required endotracheal intubation and mechanical ventilation for longer than one week and were included in the study. Tracheal aspirates were collected through endotracheal tubes into DeLee traps under sterile conditions every three days for as long as infants remained ventilated. ${ }^{6}$ A Gram stain was performed on all aspirates for the identification of bacteria and measurement of the white blood cell count. White cells were counted from 20 high power fields and the average taken. All aspirates were plated immediately onto blood, chocolate, and MacConkey agars. Plates were examined daily and organisms identified by routine laboratory techniques. ${ }^{78}$ Antibiotic sensitivity testing was done on all Gram negative bacilli and coagulase positive staphylococci that were isolated. Infants suspected of having a septic focus on the basis of clinical findings had blood from at least one percutaneous puncture site inoculated into tryptic soy broth. Antibiotic sensitivity testing was done on all 
organisms isolated from blood cultures. Over the year of study, all infants requiring ventilation received antibiotics for the first three days of life. Thereafter, antibiotics were given only during episodes of suspected or proved sepsis.

The pattern of respiratory tract colonisation over time in infants who developed sepsis was compared with that of infants who did not. Additionally, for infants who developed sepsis, correlation between blood pathogens and isolates obtained from endotracheal tubes during the week before the episode of sepsis was examined. The following definitions were used: full agreement when the organism strain isolated from blood matched the only isolate from the endotracheal tube; partial agreement when the organism strain isolated from the blood matched one of several isolates from the endotracheal tube; and no agreement when the organism found in the blood did not match any of those isolated from the endotracheal tube.

Differences in the characteristics between infants with and without sepsis were evaluated by the two tailed Student's $t$ test. Differences in the Gram stain white cell response were compared by Fisher's exact probability test. All values are expressed as mean (SEM).

\section{Results}

The study group comprised 54 boys and 40 girls who required endotracheal intubation and mechanical ventilation for a mean of 47 (5) days (range 8 to 276). All but three infants were intubated and ventilated on the first day of life. The most common reason for ventilation was respiratory distress syndrome, which occurred in $63(67 \%)$ of the infants. During the study period 26 episodes of sepsis occurred in 22 of the ventilated infants. These 22 infants with sepsis differed from the remaining 72 ventilated infants who never developed sepsis in that they weighed less at birth (926 (116) compared with 1410 (118) g, p < 0.01), were of lower gestational age (27.5 (0.7) compared with $29.6(0.6)$ weeks, $\mathrm{p}<0.05)$, and required mechanical ventilation for a longer period of time (83 (15) compared with 36 (4) days, $\mathrm{p}<0.001)$. The mean (SEM) age of infants when bacteraemia was diagnosed was 41 (7) days, range $8-130$.

A total of 1448 cultures of endotracheal tube aspirates were done on the entire group of 94 infants; $395(27 \%)$ grew no organisms, $410(28 \%)$ grew mixed Gram positive flora (including coagulase negative staphylococci, $\alpha$ haemolytic streptococci, and diphtheroids) and the remaining 643 cultures $(45 \%)$ grew one or more Gram negative bacilli, coagulase positive staphylococci, or other pathogens.

Bacterial colonisation of endotracheal tube aspirates changed similarly with the duration of ventilation in infants who developed sepsis and in those who did not (fig 1). In both groups of infants, more than $90 \%$ of the aspirates (177) obtained during the first week of ventilation were sterile. Between the second and fourth weeks of ventilation, respiratory colonisation with mixed Gram positive flora developed, and after four weeks of ventilation Gram negative bacilli predominated. Klebsiella pneumoniae, Pseudomonas species, Enterobacter species, and Escherichia coli were not isolated

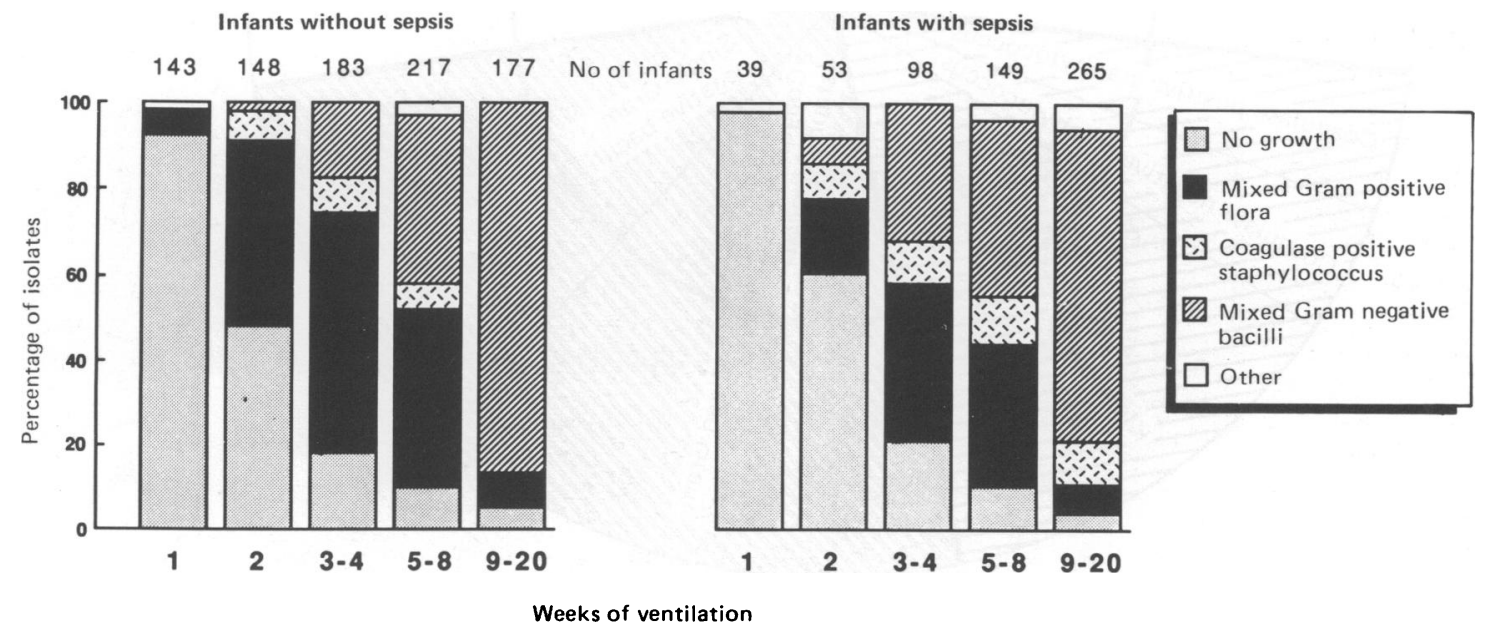

Fig 1 Developmental pattern of bacterial colonisation of endotracheal tube aspirates in infants with and without sepsis. 
during the first week of ventilation but accounted for 138 (almost $40 \%$ ) of all isolates obtained during the second month of ventilation and 316 (over $70 \%$ ) of all isolates obtained after two months of ventilation.

In the infants with sepsis, pathogens isolated from blood were compared with endotracheal tube isolates obtained during the week before the onset of clinical infection (fig 2). In 14 of the 26 cases of sepsis $(54 \%)$, the strain of organism isolated from blood was not found in the endotracheal tube aspirate. These cases of 'no agreement' included five instances in which the previous endotracheal tube aspirates had been sterile and nine instances in which
Outer ring= Isolates from endotracheal tube Inner ring = Isolates from blood
No agreement between isolates from blood and endotracheal tube

Partial agreement between isolates from blood and endotracheal tube

Full agreement between isolates from blood and endotracheal tube

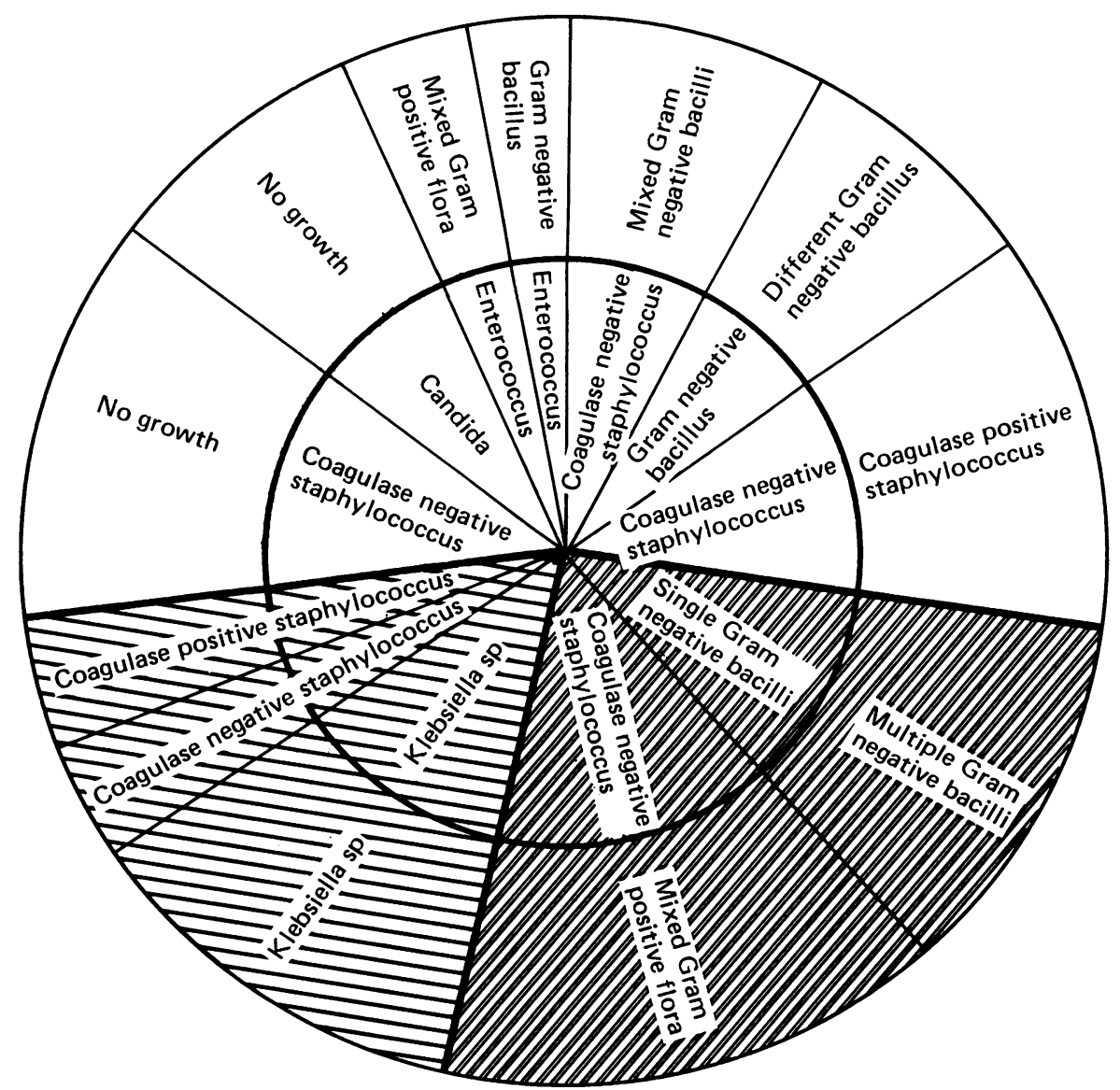

Fig2 Agreement between blood pathogens isolated during episodes of sepsis and bacteria isolated fromendotracheal tube aspirates during the previous week. 
different organisms were isolated from the endotracheal tube aspirates. In seven of the 26 cases of sepsis $(27 \%)$, the organism in the blood was found as one of multiple isolates from the endotracheal tube (partial agreement). Finally, in only five cases of sepsis $(19 \%)$ was the same single strain of organism isolated from both blood and endotracheal tube aspirate cultures (full agreement).

The length of time before an episode of sepsis that any single organism was grown from the endotracheal tube aspirate was compared in the three agreement groups. The infants with full agreement and those with partial agreement had their blood pathogen identified in endotracheal tube aspirates for a mean 17 (3) days and 11 (5) days before the onset of sepsis, respectively. These durations of endotracheal tube colonisation with a single organism before the diagnosis of sepsis did not differ significantly from that seen in infants with no correlation between blood and endotracheal tube isolates (10 (3) days).

The organisms responsible for sepsis in the ventilated infants were similar to organisms isolated from blood in 23 non-ventilated infants who developed sepsis after 1 week of age during the one year study period. Coagulase negative staphylococci were the most frequent pathogens found in the blood in both groups, accounting for $50 \%$ of positive blood cultures in ventilated infants and $11(48 \%)$ of those in non-ventilated infants. Klebsiella was the next most common pathogen and was responsible for $23 \%$ and $17 \%$ of cases of sepsis in ventilated and non-ventilated infants, respectively.

The endotracheal tube aspirate white blood cell response seen in infants during the week before an episode of sepsis was similar to that seen in aspirates taken from infants who never developed sepsis. Only four of 73 aspirates $(5 \%)$ obtained from infants who developed sepsis had more than 10 white cells/high power field while 74 of 818 aspirates (9\%) obtained from infants who never developed sepsis had a similar response. None of the Gram stains of aspirates taken from infants with full agreement between blood and endotracheal tube pathogens had this degree of white cell response.

Finally, if antibiotic choices had been based on the sensitivity of the predominant isolate from the culture of the endotracheal tube aspirates obtained just before the manifestations of sepsis there would have been inappropriate antibiotic coverage for the pathogen in the blood in 11 of the 26 cases $(42 \%)$.

\section{Discussion}

Bacterial colonisation of the respiratory tract changed with time in neonates requiring prolonged endo- tracheal intubation and mechanical ventilation. Of the endotracheal tube aspirate cultures obtained from infants during the first seven days of ventilation, $90 \%$ were sterile. The lack of organisms was probably the result of administration of antibiotics to all infants during the first three days of life. Harris et $a l^{3}$ found a significant decrease in respiratory tract colonisation in the first several days of life in ventilated infants treated with antibiotics compared with similar infants who received no antibiotics. In our population, after the first week of ventilation growths of mixed Gram positive flora emerged, and after a month of ventilation growths of Gram negative bacilli predominated. This pattern of respiratory tract colonisation was similar in infants who subsequently developed sepsis and in those who remained asymptomatic.

The value of the body surface colonisation pattern as a predictor of pathogens responsible for episodes of neonatal sepsis has been questioned. Evans $e a^{2}$ obtained cultures from 3371 infants admitted to a single newborn intensive care unit to determine how frequently isolates from body fluid cultures, primarily blood, matched those seen on previous body surface cultures. A total of 1935 infants had at least one surveillance culture of skin, mucous membrane, or gastric or endotracheal tube aspirate done during the two week period before an episode of suspected sepsis. Although agreement between the results of cultures of body fluid and surface isolates improved the nearer the time the body surface cultures were obtained to an episode of proved sepsis, the maximal sensitivity of all surface cultures was only $56 \%$ while overall specificity was $82 \%$. When the surveillance cultures of endotracheal tube aspirates were analysed separately, the sensitivity and specificity were $26 \%$ and $74 \%$, respectively. These investigators studied a heterogeneous group of infants, and results of surface cultures reflected isolates acquired from both perinatal and nosocomial exposure. Our study was designed to evaluate whether identifying respiratory tract colonisation aided the management of ventilated neonates at high risk of hospital acquired sepsis.

Previous studies examining the association between respiratory tract colonisation and systemic infection have included both non-ventilated infants and infants ventilated for brief periods of time. Sprunt et al ${ }^{9}$ prospectively monitored oropharyngeal colonisation in 223 nursery admissions between 1971 and 1974. In that study none of the 156 infants colonised with $\alpha$ haemolytic streptococci or other bacteria in low titre developed sepsis. In contrast, 14 of 67 infants colonised with potential pathogens in high titre developed systemic infection. In almost every case these infections were caused by colonising 
bacteria. The effects of endotracheal intubation and mechanical ventilation on respiratory colonisation or infection were not evaluated.

Harris $\mathrm{et}^{\mathrm{al}} \mathrm{l}^{3}$ prospectively studied 54 neonates who required orotracheal intubation. Respiratory tract colonisation was monitored with nasopharyngeal as well as tracheal aspirate cultures. The respiratory tracts of 23 infants were already colonised at the time of intubation; nine of the infants $(39 \%)$ were concurrently infected. Sixteen additional infants subsequently developed respiratory tract colonisation and eight of those $(50 \%)$ developed sepsis. In contrast, none of the 10 infants who remained uncolonised developed sepsis. As more than $80 \%$ of all infants were intubated for less than three days the correlation between respiratory tract flora and organisms isolated from blood may have reflected congenital infections. Sherman et $a l^{4}{ }^{10}$ provided data showing that tracheal aspirates obtained within the first eight hours after birth were reliable in establishing the diagnosis of congenital pneumonia and bacteraemia. The authors pointed out, however, that the presence of bacteria in tracheal secretions lost its diagnostic usefulness after the infant was more than 24 hours old when the normally sterile fetal lung becomes colonised. Most ventilated infants in our series who developed sepsis after one week of life showed no correlation between pathogens isolated from blood and previous endotracheal tube isolates. Other putative markers for infection such as endotracheal tube aspirate white cell response and changes in respiratory tract bacterial flora were also unhelpful in identifying infants who subsequently developed sepsis. The part played by serial cultures of endotracheal tube aspirates in the diagnosis of hospital acquired infections that are limited to the respiratory tract (such as tracheitis and pneumonia) remains to be elucidated.

The use of routine surveillance cultures other than those directed at tracing a specific organism during an epidemic ${ }^{11}$ has been likened to an exercise in futility. ${ }^{12}$ Though serial cultures of endotracheal tube aspirates may be useful in establishing the general nursery flora, their limited value to an individual infant does not justify routine surveillance in all ventilated infants.

We thank Myrna Pantangco and Pat Hammel for their help in preparing this manuscript.

\section{References}

1 Sprunt K. Practical use of surveillance for prevention of nosocomial infection. Semin Perinatol 1985;9:47-50.

2 Evans ME, Schaffner W, Federspiel CF, Cotton RB, McKee KT, Stratton CW. Sensitivity, specificity, and predictive value of body surface cultures in a neonatal intensive care unit. $J A M A$ 1988;259:248-52.

${ }^{3}$ Harris H, Wirtschafter D, Cassady G. Endotracheal intubation and its relationship to bacterial colonization and systemic infection of newborn infants. Pediatrics 1976;56:816-23.

${ }^{4}$ Sherman MP, Goetzman BW, Ahlfors CE, Wennberg RP. Tracheal aspiration and its clinical correlates in the diagnosis of congenital pneumonia. Pediatrics 1980;65:258-63.

${ }^{5}$ Brook I, Martin WJ, Finegold SM. Bacteriology of tracheal aspirates in intubated newborn. Chest 1980;78:875-7.

6 Gregory GA. Respiratory care of newborn infants. Pediatr Clin North Am 1971;19:311-24.

${ }^{7}$ Lennette EH, Balows A, Hausler WJ, et al. Manual of clinical microbiology. Washington: American Society for Microbiology, 1985.

${ }^{8}$ Isenberg HD, Schoenknecht FD, von Graevenitz A. Cumulative techniques: collection and processing of bacteriologic specimens. Washington: American Society for Microbiology, 1979.

9 Sprunt K, Leidy G, Redman W. Abnormal colonization of neonates in an intensive care unit: means of identifying neonates at risk of infection. Pediatr Res 1978;12:998-1002.

10 Sherman MP, Chance KH, Goetzman BW. Gram's stains of tracheal secretions predict neonatal bacteremia. Am J Dis Child 1984;138:848-50.

$"$ Smith DH. Epidemics of infectious diseases in newborn nurseries. Clin Obstet Gynecol 1979;22:409-23.

12 Fulginiti VA. Body surface cultures in the newborn infant. An exercise in futility, wastefulness and inappropriate practice. Am J Dis Child, 1988,142:19-20.

Correspondence and requests for reprints to Dr. TA Slagle, Department of Neonatology, Children's Hospital of San Francisco, 3700 California Street, San Francisco, CA 94118, USA.

Accepted 26 September 1988. 